\title{
Observation of electron cyclotron harmonic emissions due to electrostatic instabilities in mirror-confined plasma
}

\author{
B. Eliasson $\odot,{ }^{1},{ }^{*}$ M. Viktorov $\odot,{ }^{2}$ D. C. Speirs, ${ }^{1}$ K. Ronald,,${ }^{1}$ D. Mansfeld,${ }^{2}$ and A. D. R. Phelps $\odot^{1}$ \\ ${ }^{1}$ SUPA, Department of Physics, University of Strathclyde, John Anderson Building, Glasgow G4 ONG, United Kingdom \\ ${ }^{2}$ Institute of Applied Physics of the Russian Academy of Sciences, 46 Ulyanov Street, 603950 Nizhny Novgorod, Russia
}

(Received 15 July 2020; accepted 4 November 2020; published 23 November 2020)

\begin{abstract}
Electron cyclotron instabilities are of fundamental importance in space and laboratory plasmas having nonthermal electron distribution functions. Observations are reported of electromagnetic emissions at electron cyclotron harmonics in a mirror-confined electron cyclotron resonance plasma. The emissions are attributed to electrostatic instabilities involving a warm $200-400 \mathrm{eV}$ mirror-confined population of electrons and a much colder $\sim 1 \mathrm{eV}$ component confined by the positive plasma potential. Due to electron-ion Coulomb collisions, electrons with energy $\lesssim 50 \mathrm{eV}$ are scattered into the loss cone, leading to a warm ring distribution with depleted low-energy components. The combination of a warm ring distribution and a cold distribution is susceptible to electrostatic cyclotron instabilities, with the unstable modes having frequencies near the upper hybrid frequency of the cold component, which can be significantly lower than the overall upper hybrid frequency. Coulomb collisions are shown to be capable of forming the ring distribution for typical experimental parameters and a linear stability analysis is undertaken using a model thermal ring distribution with different fractions of cold electrons. The observations are supported by Vlasov simulations that are used to study the nonlinear evolution of the instabilities.
\end{abstract}

DOI: 10.1103/PhysRevResearch.2.043272

\section{INTRODUCTION}

Electron cyclotron instabilities are of great importance for the wave-particle dynamics and overall energy balance in the near-Earth plasma environment [1], while in the solar wind, cyclotron instabilities limit the particle distribution function and put a bound on the electron temperature anisotropy $[2,3]$. The auroral kilometric radiation (AKR) is attributed to electromagnetic instability due to electrons compressed in the converging magnetic field of planetary auroral regions, yielding horseshoe distributions giving maser emission into intense $\mathrm{X}$-mode radiation [4], while similar emissions are observed to occur in other astrophysical environments [5,6]. Electron anisotropy gives rise to the electromagnetic electron cyclotron (whistler) instability with frequencies below the electron cyclotron frequency, when the perpendicular temperature is larger than the parallel temperature [7-9]. Electrostatic waves play an important role in the scattering of the hot population of magnetospheric electrons, which leads to its precipitation into the atmosphere and the excitation of diffuse auroral emissions at the Earth [10,11] and other planets [12]. There are recent space-borne observations at the Earth's bow shock and

\footnotetext{
*Author to whom correspondence should be addressed: bengt.eliasson@strath.ac.uk

Published by the American Physical Society under the terms of the Creative Commons Attribution 4.0 International license. Further distribution of this work must maintain attribution to the author(s) and the published article's title, journal citation, and DOI.
}

magnetosphere of electrostatic waves with frequencies at electron cyclotron harmonics [13-15] and half harmonics [16-18], which are attributed to electrostatic electron cyclotron instabilities due to ringlike electron velocity distribution functions. Electron cyclotron waves are inherent not only to the Earth, but are also found in the magnetospheres of Jupiter [19,20] and Saturn [21,22].

Led by early space and laboratory observations of electron cyclotron emissions, theoretical works on cyclotron instabilities due to cold velocity ring distributions were initiated in the 1960s and 1970s [23-26] and in more recent works on thermal ring distributions [27]. The inclusion of a minority cold core distribution lowers the threshold for the instability for more realistic distributions having warmer ring populations, as found in theory [28,29], space observations [30], and simulations [31-34]. The instability preferably takes place with frequencies near the upper hybrid frequency of the cold component $[29,30]$, and hence the spectra of unstable waves can be used to estimate the density of the cold component which otherwise can be difficult to measure.

Understanding the broad range of space plasma environments where the excitation of cyclotron emissions is observed benefits from laboratory investigations under similar plasma conditions. In previous laboratory experiments and associated numerical simulations, cyclotron emissions from half-shell distributions relevant to AKR phenomena were studied [35-37], while electron Bernstein waves were triggered by antennas or by the injection of electron beams into a preexisting plasma [38-42]. The present experiment is based on another approach, in which plasma waves are generated by the unstable electron velocity distribution of 


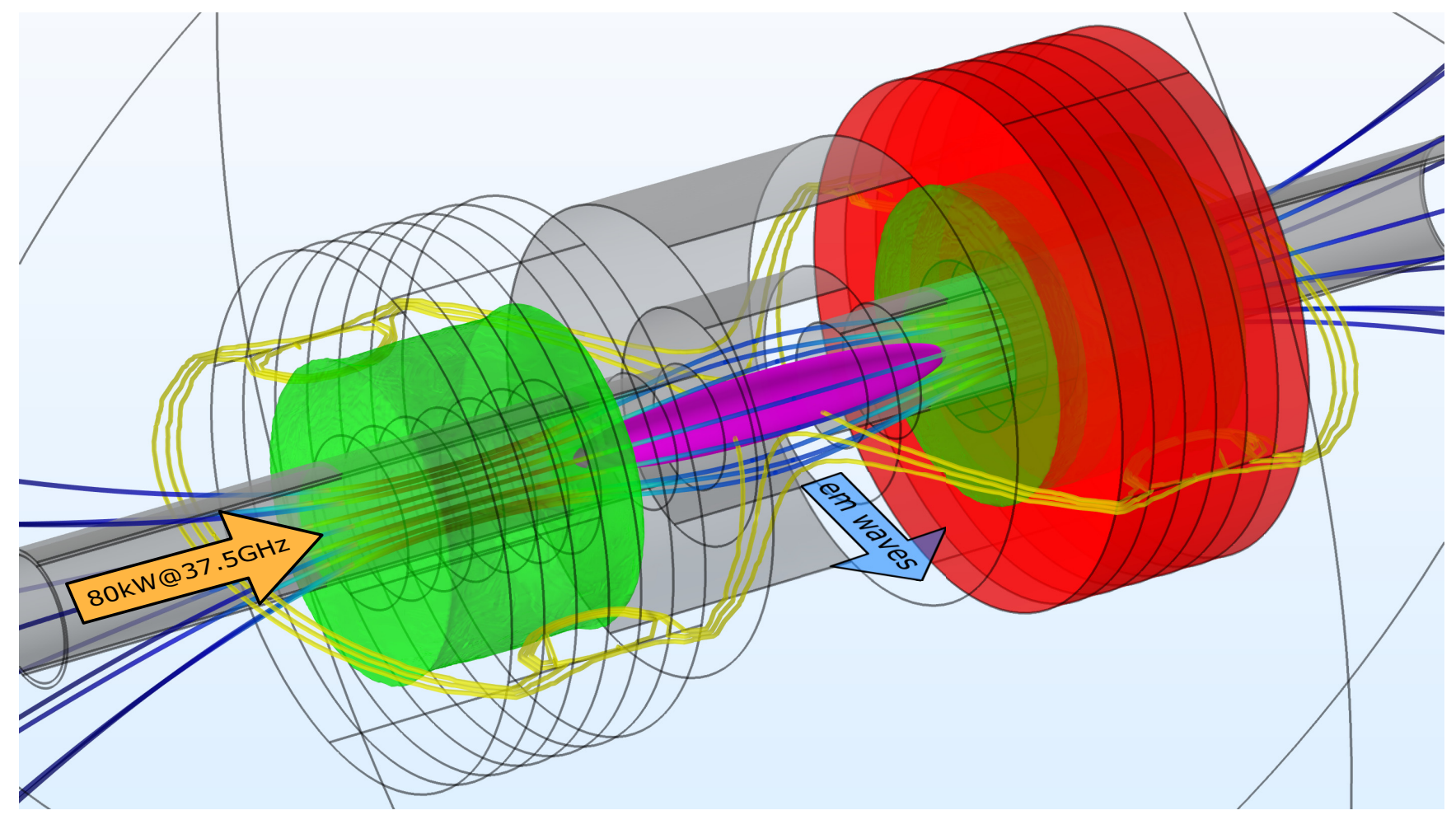

FIG. 1. A schematic view of the experiment design, showing the vacuum chamber (gray) and magnetic coils (red). Microwave radiation is launched along the trap axis (orange arrow) and creates plasma (purple volume), which is confined in a mirror magnetic trap (magnetic field lines are shown in blue). ECR surfaces are shown in green, and several isolines of constant magnetic field in a plane cross section are shown in yellow. Blue arrow denotes microwave emissions by the plasma, studied in the present experiment.

a core plasma. In the present setup using a simple mirror trap $[43,44]$, experiments of plasma created and sustained by high-power gyrotron microwave radiation under the electron cyclotron resonance (ECR) condition have demonstrated the existence of a warm dense mirror-contained electron component having a temperature of $200-400 \mathrm{eV}$ and a less dense component of hot electrons with an anisotropic distribution function and an average energy of about $10 \mathrm{keV}$, which is typical for the ECR discharges $[45,46]$. The fraction of hot electrons is estimated to be on the order of $10^{-2}$. There are also observations of a very hot tail of electrons with relativistic energies $100-400 \mathrm{keV}[47,48]$. In addition, previous studies of other ECR discharges [49] have also shown the existence of a minority of cold electrons that are confined by the positive plasma potential. Hence, the mirror ECR plasma may contain electron distributions similar to the ones observed in space, involving warm and hot components that are anisotropic and constitute the free energy for cyclotron instability, and a cold component that enables the instability and controls the wave frequency for which the instability takes place. An alternative candidate for instability, the electron cyclotron drift instability [50], would require that the $\mathbf{E} \times \mathbf{B}$ drift speed $v_{\mathbf{E} \times \mathbf{B}}=\mathbf{E} \times$ $\mathbf{B} / c B^{2}$ exceed the electron thermal speed $v_{\mathrm{T}}$. In our experiment [51] this is however not the case with $v_{\mathbf{E} \times \mathbf{B}} \approx 10^{6} \mathrm{~cm} / \mathrm{s}$ and $v_{\mathrm{T}} \approx 10^{8} \mathrm{~cm} / \mathrm{s}$.

The aim of this paper is to use the results of theoretical analysis and simulations to understand recent experimental measurements [44] of electromagnetic emission spectra. During the developed discharge stage with $\omega_{\text {pe }} \gg \omega_{\text {ce }}$, electromagnetic emissions were observed at frequencies near harmonics of the electron cyclotron frequency, which may now be interpreted as due to the excitation of electron Bernstein waves. The theoretical analysis suggests that the warm mirror-trapped component with a mean energy in the range 200-400 eV coexists with a much cooler component with mean energy on the order of $1 \mathrm{eV}$, which is electrostatically trapped by the positive potential and effectively tied to the ions also having a temperature of $\sim 1 \mathrm{eV}$. Due to the Coulomb collisions with ions, the warmer component is depleted for energies less than about $50 \mathrm{eV}$, leading to the formation of a warm ring distribution. As mentioned above, the combination of a warm ring distribution and a cold component is susceptible to electrostatic electron cyclotron instabilities at frequencies near the upper hybrid frequency calculated from the number density of the less dense cold component. There is significant free energy in the denser warm ring distribution that can be released through instability and lead to radiation of electromagnetic waves. The instability is here investigated by means of linear stability analysis based on the dispersion relation for model distributions, and Vlasov simulations to capture the nonlinear behavior of the system.

\section{EXPERIMENTAL SETUP}

The experiment was conducted with a plasma sustained through ECR heating by the radiation output of a gyrotron (frequency $37.5 \mathrm{GHz}$, power up to $80 \mathrm{~kW}$, pulse duration $1 \mathrm{~ms}$ ) in an axially symmetric open magnetic trap [51]. The design of the experiment is schematically shown in Fig. 1. Microwave radiation is launched from the gyrotron into the discharge 
chamber along the trap axis through a plasma matching device. The matching element has the shape of a wedge firmly connected to the vacuum chamber, which ensures the passage of more than $90 \%$ of the microwave radiation power into the discharge chamber. The radiation intensity in the focal plane is about $10 \mathrm{~kW} / \mathrm{cm}^{2}$ and the average power density is $100 \mathrm{~W} / \mathrm{cm}^{3}$.

The discharge chamber is placed in a mirror magnetic trap, produced by pulsed coils with a maximum magnetic field strength of $4.3 \mathrm{~T}$, and a pulse duration of about $7.3 \mathrm{~ms}$. The length of the magnetic trap is $22.5 \mathrm{~cm}$ with the mirror ratio $R=B_{\max } / B_{\min } \approx 5$. The value of the magnetic field strength in the trap as a function of space and time is defined by the charging voltage on the magnetic field system capacitor bank and the relative delay from the beginning of the magnetic field pulse. Varying these two parameters determines the absolute value of the magnetic field and its time derivative.

The plasma is created and supported under ECR conditions at the fundamental cyclotron harmonic at $37.5 \mathrm{GHz}$ corresponding to a magnetic field strength of $1.34 \mathrm{~T}$. The resonance surface is situated between the magnetic mirror and the center of the discharge chamber. The ambient pressure of the neutral gas (nitrogen) is about $10^{-6}$ Torr; however it increases up to $10^{-4}$ to $10^{-3}$ Torr by gas puffing during the ECR discharge. The injection of the working gas is carried out through the inlet channel located inside the plasma matching device, which is placed in a magnetic mirror plug. In addition, the metal surface of the matching element is also used as a plasma limiter. Therefore, plasma does not form in the alternate ECR zone outside the mirror trap.

The dynamic evolution of the spectrum and intensity of the emitted electromagnetic radiation from the plasma are studied with the use of two different receivers: a broadband horn antenna with a flat spectral response in the range 2-20 GHz (input aperture $104 \times 78 \mathrm{~mm}^{2}$ ), which is used together with an antialias low-pass filter with $30 \mathrm{~dB}$ rejection for frequencies exceeding $24.66 \mathrm{GHz}$, and the open face of a waveguide filter with passband $27.0-36.0 \mathrm{GHz}$ (input aperture $7.2 \times 3.4 \mathrm{~mm}^{2}$ ) feeding a waveguide-coaxial transition. Signals are recorded by a high-performance oscilloscope Keysight DSA-Z 594A (analog bandwidth $59 \mathrm{GHz}$, sampling rate $160 \mathrm{GSample} / \mathrm{s}$ ). In the scope of the experiment, the antenna bandwidths cover frequencies up to the fifth harmonic of the electron gyrofrequency $f_{\text {ce }}$ at the trap center. The dynamic spectra are calculated from the recorded data by short-time Fourier transforms windowed with a Hamming window. Simultaneously, we measure the precipitation of energetic electrons (10-180 keV) from the trap ends using a $p$ - $i$ - $n$ diode detector with a time resolution of about 1 ns [52].

\section{OBSERVATION OF ELECTROMAGNETIC EMISSIONS AT CYCLOTRON HARMONICS}

In the experiment, the developed dense plasma discharge stage is considered, lasting from the ECR breakdown until the end of the microwave heating pulse with a duration of $1 \mathrm{~ms}$. At that stage an electron population has been formed containing one warm dense component $\left(n_{\mathrm{w}} \sim 10^{13} \mathrm{~cm}^{-3}\right.$, mean energy $E_{\mathrm{w}} \sim 300 \mathrm{eV}$ ) and a less dense component of hot electrons $\left(n_{\mathrm{h}} \sim 10^{11} \mathrm{~cm}^{-3}\right.$, mean energy $\left.E_{\mathrm{h}} \sim 10 \mathrm{keV}\right)$
$[43,44,52]$. Our theoretical analysis below also suggests the existence of a third cold component $\left(n_{\mathrm{c}} \sim 10^{12}-10^{13} \mathrm{~cm}^{-3}\right.$, temperature $T_{\mathrm{c}} \sim 1 \mathrm{eV}$ ).

During the developed discharge stage the diagnostics simultaneously registered plasma microwave emissions of two types: low-frequency emissions at frequencies about $f_{\text {ce }} / 2$ attributed to an electromagnetic electron cyclotron instability [51], and high-frequency emissions at frequencies in the range $1-5 f_{\text {ce }}$. Here the analysis focuses on the latter type of microwave radiation. An example of such an instability event is shown in Fig. 2. Microwave emissions are observed in frequency bands that follow certain cyclotron harmonics, while the ambient magnetic field is slowly increasing with time. Interestingly, emissions are first seen at frequencies between $3 f_{\text {ce }}$ and $4 f_{\text {ce }}$ and weaker emissions between $4 f_{\text {ce }}$ and $5 f_{\text {ce }}$, and at a later stage emissions are instead observed at $2 f_{\text {ce }}$, indicating a gradual evolution of the plasma parameters in time. Emissions near $4 f_{\text {ce }}$ are identified as nonlinear harmonics of simultaneous emissions at $2 f_{\text {ce. }}$. Assuming that the total electron number density $n_{0}=n_{\mathrm{c}}+n_{\mathrm{w}}+n_{\mathrm{h}}$ corresponds to the critical density for the gyrotron radiation at $37.5 \mathrm{GHz}$ and equals $n_{0}=1.7 \times 10^{13} \mathrm{~cm}^{-3}$, one may estimate the value of the upper hybrid resonant frequency $\omega_{\mathrm{uh}}=\sqrt{\omega_{\mathrm{pe}}^{2}+\omega_{\mathrm{ce}}^{2}}$ during the instability onset. Here $\omega_{\mathrm{pe}}=\sqrt{4 \pi n_{0} e^{2} / m_{\mathrm{e}}}$ is the electron plasma frequency, $\omega_{\mathrm{ce}}=e B_{0} / m_{\mathrm{e}} c$ is the electron cyclotron frequency, where $B_{0}$ is the magnetic field strength, $e$ and $m_{\mathrm{e}}$ are the unit charge and the electron mass, respectively, and $c$ is the speed of light in vacuum. Under the experimental conditions in Fig. 2, the ratio $\omega_{\mathrm{uh}} / \omega_{\mathrm{ce}}$ decreases from 5.1 to 4.5 due to the increase of the electron cyclotron frequency from $7.5 \mathrm{GHz}$ to $8.6 \mathrm{GHz}$. The value $\omega_{\mathrm{uh}} / \omega_{\mathrm{ce}}=5.1$ will be used in the theoretical and numerical calculations below.

It was noted [44] that the intensity of the recorded cyclotron harmonic emissions do not correlate with precipitations of hot electrons from the trap ends. In contrast, low-frequency emissions in the whistler range at frequencies about $f_{\mathrm{ce}} / 2$ are accompanied by intense precipitations of hot electrons [51].

\section{COLLISIONAL DIFFUSION INTO THE LOSS CONE}

A viable mechanism for the formation of a warm electron ring distribution in velocity space is the loss of low-velocity electrons by Coulomb collisions with ions. The electron-ion Coulomb collision frequency for an electron moving with speed $v$ is $v_{\mathrm{ei}}=n_{\mathrm{i}} \sigma_{\mathrm{ei}} v$, where the collision cross section for small-angle collisions can be estimated as [53]

$$
\sigma_{\mathrm{ei}}=\frac{4 \pi Z_{\mathrm{i}}^{2} e^{4} \ln \Lambda}{m_{\mathrm{e}}^{2} v^{4}},
$$

where $Z_{\mathrm{i}}$ is the ion charge state. The cross section is strongly dependent on $v$. The corresponding mean-free path is

$$
\lambda_{\mathrm{mfp}}=\frac{v}{v_{\mathrm{ei}}}=\frac{1}{n_{\mathrm{i}} \sigma_{\mathrm{ei}}}=\frac{m_{\mathrm{e}}^{2} v^{4}}{4 \pi n_{\mathrm{i}} Z_{\mathrm{i}}^{2} e^{4} \ln \Lambda} .
$$

A reasonable approximation of the Coulomb logarithm is $\ln \Lambda \approx 15$. 

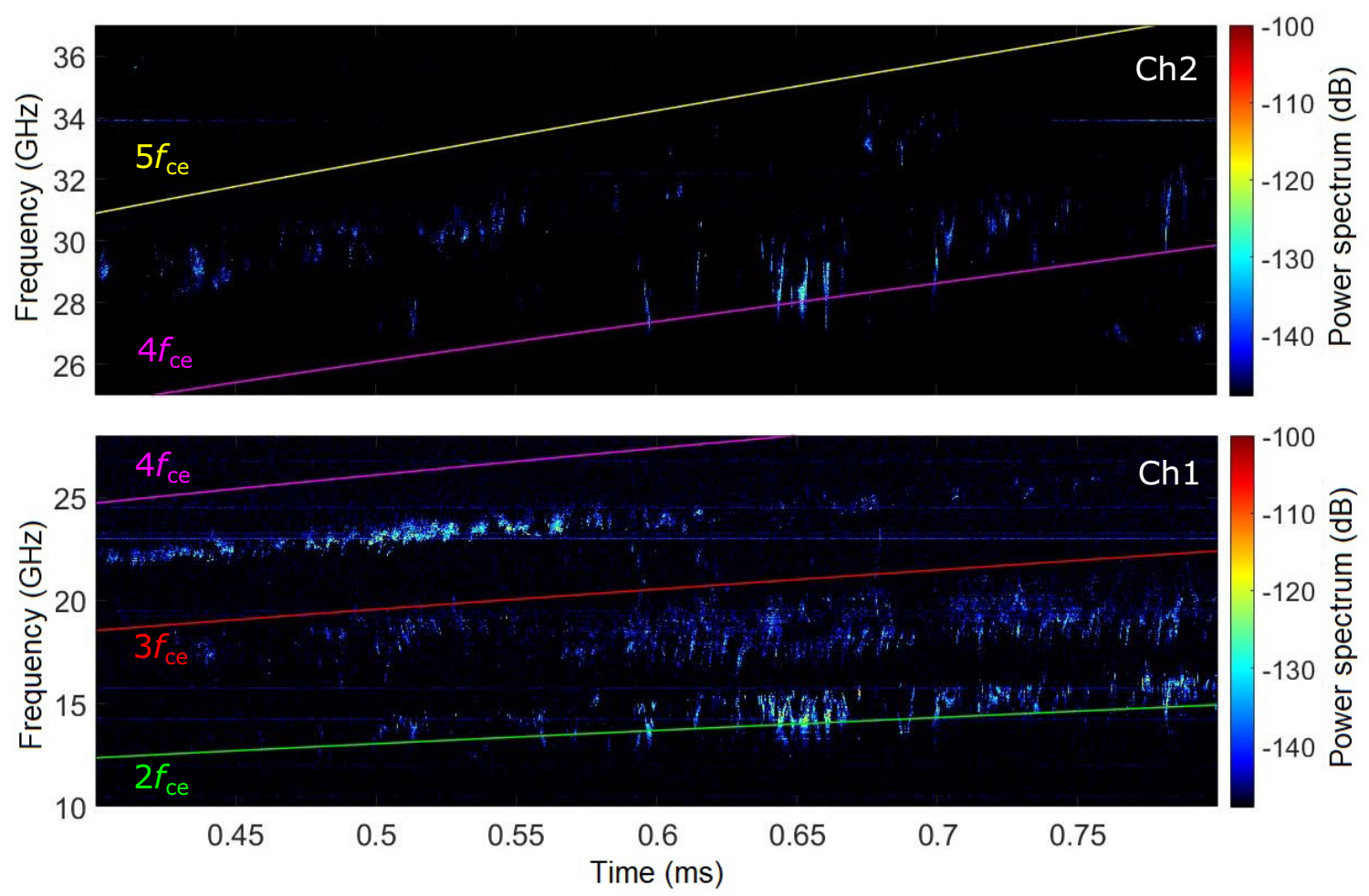

FIG. 2. Dynamic spectrum of plasma microwave emissions during the ECR discharge recorded simultaneously by two independent antennas. The frequencies of different electron cyclotron harmonics at the trap center are indicated by the solid lines on the dynamic spectrum. The time reference starts at the moment the gyrotron is turned on, and the gyrotron radiation turns off after $1 \mathrm{~ms}$.

The electrons can be considered collision-free if $\lambda_{\operatorname{mfp}} \gg L$, where $L$ is the length of the plasma, giving

$$
\frac{v}{v_{\mathrm{ei}}}=\frac{m_{\mathrm{e}}^{2} v^{4}}{4 \pi n_{\mathrm{i}} Z_{\mathrm{i}}^{2} e^{4} \ln \Lambda} \gg L
$$

Solving for $v$ the condition $v>v_{*}$ is obtained, where (in $\mathrm{cm} / \mathrm{s})$

$$
v_{*}=\left(\frac{4 \pi L n_{\mathrm{i}} Z_{\mathrm{i}}^{2} e^{4} \ln \Lambda}{m_{\mathrm{e}}^{2}}\right)^{1 / 4} \approx 5.9 \times 10^{4}\left(L n_{\mathrm{i}} Z_{\mathrm{i}}^{2}\right)^{1 / 4} .
$$

In a high-power ECR discharge, the formation of multiply charged ions is highly probable. Based on previous experience for the same setup [54,55], the average ion charge number in the present experiment is estimated to be in the range $Z_{\mathrm{i}}=2-4$. Estimates of $v_{*}$ and the corresponding values of the electron kinetic energy $W_{\mathrm{k}}^{*}=m_{\mathrm{e}} v_{*}^{2} / 2$ are shown in Table I for parameters relevant to the experiment. Thus, electrons with energies above $\sim 50 \mathrm{eV}$ may be considered to be collisionless, while electrons with lower energies suffer collisions and can

TABLE I. Limiting electron velocity $v_{*}$ and corresponding kinetic energy $W_{\mathrm{k}}^{*}$ for electrons to be collisionless.

\begin{tabular}{cccccc}
\hline \hline & $\begin{array}{c}n_{\mathrm{e}} \\
\left(\mathrm{cm}^{-3}\right)\end{array}$ & $\begin{array}{c}n_{\mathrm{i}}=n_{\mathrm{e}} / Z_{\mathrm{i}} \\
\left(\mathrm{cm}^{-3}\right)\end{array}$ & $\begin{array}{c}L \\
(\mathrm{~cm})\end{array}$ & $\begin{array}{c}v_{*} \\
(\mathrm{~cm} / \mathrm{s})\end{array}$ & $\begin{array}{c}W_{\mathrm{k}}^{*} \\
(\mathrm{eV})\end{array}$ \\
\hline 1 & $10^{13}$ & $10^{13}$ & 20 & $2.2 \times 10^{8}$ & 14 \\
4 & $10^{13}$ & $2.5 \times 10^{12}$ & 20 & $3.1 \times 10^{8}$ & 28 \\
7 & $10^{13}$ & $1.4 \times 10^{12}$ & 20 & $3.6 \times 10^{8}$ & 37 \\
\hline \hline
\end{tabular}

be scattered into the loss cone and be lost. The remaining electrons with a temperature $\sim 200-400 \mathrm{eV}$ will form a thermal ring distribution with depleted low-energy components.

Through the loss of electrons with energies less than $50 \mathrm{eV}$ into the loss cone, the plasma becomes overall positively charged and a positive potential develops. A population of cold electrons with energies $\ll 50 \mathrm{eV}$ may be electrostatically trapped in the positive potential and tied to the ions. Through frequent collisions, the temperature of the cold electron component approaches thermal equilibrium with the ions with a temperature of $\sim 1 \mathrm{eV}$. Such combinations of warm and cold electrons have been observed in past ECR plasma experiments [49]. Hence, in the present experiment there is the possibility of a multicomponent plasma with a warm thermal ring distribution with a temperature of $\sim 200-400 \mathrm{eV}$ and a cold, Maxwellian distribution with a temperature of $\sim 1 \mathrm{eV}$, which is susceptible to electrostatic electron cyclotron instabilities. The significantly smaller fraction of hot electrons with energies on the order of $10 \mathrm{keV}$ will be omitted in the calculations below.

\section{STABILITY ANALYSIS AND VLASOV SIMULATIONS}

Consider electrostatic waves propagating perpendicularly to the magnetic field $\mathbf{B}_{0}=B_{0} \widehat{\mathbf{z}}$ so that in the plane perpendicular to $\mathbf{B}_{0}$ one has $\mathbf{r}=x \widehat{\mathbf{x}}+y \widehat{\mathbf{y}}$ and $\nabla=\widehat{\mathbf{x}} \partial / \partial x+\widehat{\mathbf{y}} \partial / \partial y$, where $\widehat{\mathbf{x}}, \widehat{\mathbf{y}}$, and $\widehat{\mathbf{z}}$ are the unit vectors along the $x, y$, and $z$ axes. The electron and ion dynamics are governed by the 
two-dimensional Vlasov-Boltzmann equations for the warm and cold electrons, respectively,

$$
\begin{aligned}
& \frac{\partial f_{\mathrm{w}}}{\partial t}+\mathbf{v} \cdot \nabla f_{\mathrm{w}}-\frac{e}{m_{\mathrm{e}}}\left(-\nabla \phi+\frac{\mathbf{v} \times \widehat{\mathbf{z}} B_{0}}{c}\right) \cdot \nabla_{\mathbf{v}} f_{\mathrm{w}} \\
& =\frac{1}{\tau_{\mathrm{w}}}\left(\eta_{\mathrm{w}} F_{\mathrm{w}}-f_{\mathrm{w}}\right), \\
& \frac{\partial f_{\mathrm{c}}}{\partial t}+\mathbf{v} \cdot \nabla f_{\mathrm{c}}-\frac{e}{m_{\mathrm{e}}}\left(-\nabla \phi+\frac{\mathbf{v} \times \widehat{\mathbf{z}} B_{0}}{c}\right) \cdot \nabla_{\mathbf{v}} f_{\mathrm{c}} \\
& =\frac{1}{\tau_{\mathrm{c}}}\left[\left(1-\eta_{\mathrm{w}}\right) F_{\mathrm{c}}-f_{\mathrm{c}}\right],
\end{aligned}
$$

and the ions

$$
\begin{aligned}
& \frac{\partial f_{\mathrm{i}}}{\partial t}+\mathbf{v} \cdot \nabla f_{\mathrm{i}}+\frac{e Z_{\mathrm{i}}}{m_{\mathrm{i}}}\left(-\nabla \phi+\frac{\mathbf{v} \times \widehat{\mathbf{z}} B_{0}}{c}\right) \cdot \nabla_{\mathbf{v}} f_{\mathrm{i}} \\
& \quad=\frac{1}{\tau_{\mathrm{i}}}\left(F_{\mathrm{i}}-f_{\mathrm{i}}\right),
\end{aligned}
$$

where $\mathbf{v}=v_{x} \widehat{\mathbf{x}}+v_{y} \widehat{\mathbf{y}}$ is the velocity variable. The system is closed by Poisson's equation for the electrostatic potential

$$
\nabla^{2} \phi=4 \pi e n_{0} \iint\left(f_{\mathrm{w}}+f_{\mathrm{c}}-Z_{\mathrm{i}} f_{\mathrm{i}}\right) d v_{x} d v_{y},
$$

where $m_{\mathrm{i}}$ is the ion mass. The electrons are continuously lost by collisions and scattering by waves, but are replaced by the ECR heating source. This is modeled by a simple tau approximation on the right-hand sides of Eqs. (5)-(7), with $\tau_{\mathrm{w}}, \tau_{\mathrm{c}}$, and $\tau_{\mathrm{i}}$ being the respective timescales for the process, and $F_{\mathrm{w}}$, $F_{\mathrm{c}}$, and $F_{\mathrm{i}}$ (defined below) represent the respective equilibrium distribution function normalized such that its integral over velocity space produces unity, and $\eta_{\mathrm{w}}=n_{\mathrm{w}} / n_{0}$ is the density fraction of warm electrons. In the numerical simulations, the values $\tau_{\mathrm{c}}=\tau_{\mathrm{w}}=500 \omega_{\mathrm{pe}}^{-1}$ and $\tau_{\mathrm{i}}=5000 \omega_{\mathrm{pe}}^{-1}$ are used. The ions do not participate significantly in the fast dynamics of electron Bernstein waves but form a neutralizing background contributing only to lower hybrid oscillations.

Small-amplitude electron Bernstein waves are found by linearizing the unknown variables and Fourier-analyzing the Vlasov-Poisson system by assuming that the first-order unknown variables are proportional to $\exp (i \mathbf{k} \cdot \mathbf{r}-i \widetilde{\omega} t)$, where $\mathbf{k}=k_{x} \widehat{\mathbf{x}}+k_{y} \widehat{\mathbf{y}}$ is the wave vector, $\widetilde{\omega}$ is the angular wave frequency, and $i=\sqrt{-1}$ is the imaginary unit. For a plasma consisting of a sum of a Maxwellian core electron distribution for the cold component and a thermal ring distribution for the warm electron component, the dispersion relation governing the linear growth rate of the instability can be written (neglecting collisions)

$$
1+\left(1-\eta_{\mathrm{w}}\right) \chi_{\mathrm{c}}+\eta_{\mathrm{w}} \chi_{\mathrm{w}}+\chi_{\mathrm{i}}=0,
$$

where $\chi_{\mathrm{c}}$ and $\chi_{\mathrm{w}}$ represent the contributions to electric susceptibilities by the cold Maxwell distributed and warm thermal ring distributed electrons, respectively, and $\chi_{\mathrm{i}}$ is the ion susceptibility.

For the warm electron component, the equilibrium distribution function is $f_{\mathrm{w}}=\eta_{\mathrm{w}} F_{\mathrm{w}}$ with a thermal ring distribution assumed to be of the form $[23,56,57]$

$$
F_{\mathrm{w}}\left(v_{\perp}\right)=\frac{v_{\perp}^{2}}{4 \pi v_{\mathrm{Tw}}^{4}} \exp \left(-\frac{v^{2}}{2 v_{\mathrm{Tw}}^{2}}\right),
$$

where $v^{2}=v_{\perp}^{2}+v_{z}^{2}$ is the electron speed squared, and $v_{\mathrm{Tw}}$ represents the thermal velocity of the warm electron component. The corresponding susceptibility is [58]

$$
\begin{aligned}
\chi_{\mathrm{w}}= & \frac{\omega_{\mathrm{pe}}^{2}}{\omega_{\mathrm{ce}}^{2}} \frac{\exp \left(-\lambda_{\mathrm{w}}\right)}{\sin (\pi \Omega)} \int_{0}^{\pi} \sin (\psi \Omega) \sin \psi \\
& \times\left[1-2 \lambda_{\mathrm{w}} \cos ^{2}(\psi / 2)\right] \exp \left(-\lambda_{\mathrm{w}} \cos \psi\right) d \psi,
\end{aligned}
$$

where $\lambda_{\mathrm{w}}=k_{\perp}^{2} v_{\mathrm{Tw}}^{2} / \omega_{\mathrm{ce}}^{2}$ and $\Omega=\omega / \omega_{\mathrm{ce}}$. For the cold electron component, the equilibrium distribution function is $f_{\mathrm{c}}=(1-$ $\left.\eta_{\mathrm{w}}\right) F_{\mathrm{c}}$ with the Maxwellian distribution function

$$
F_{\mathrm{c}}\left(v_{\perp}\right)=\frac{1}{2 \pi v_{\mathrm{Tc}}^{2}} \exp \left(-\frac{v^{2}}{2 v_{\mathrm{Tc}}^{2}}\right),
$$

where $v_{\mathrm{Tc}}=\left(k_{B} T_{\mathrm{c}} / m_{\mathrm{e}}\right)^{1 / 2}$ is the thermal speed of the cold electrons. The corresponding susceptibility is [24]

$$
\begin{aligned}
\chi_{\mathrm{c}}= & \frac{\omega_{\mathrm{pe}}^{2}}{\omega_{\mathrm{ce}}^{2}} \frac{\exp \left(-\lambda_{\mathrm{c}}\right)}{\sin (\pi \Omega)} \int_{0}^{\pi} \sin (\psi \Omega) \sin \psi \\
& \times \exp \left(-\lambda_{\mathrm{c}} \cos \psi\right) d \psi,
\end{aligned}
$$

where $\lambda_{\mathrm{c}}=k_{\perp}^{2} v_{\mathrm{Tc}}^{2} / \omega_{\mathrm{ce}}^{2}$. For the ions, the equilibrium distribution function is $f_{\mathrm{i}}=F_{\mathrm{i}}$ with the Maxwellian distribution function

$$
F_{\mathrm{i}}\left(v_{\perp}\right)=\frac{1}{2 \pi v_{\mathrm{Ti}}^{2}} \exp \left(-\frac{v^{2}}{2 v_{\mathrm{Ti}}^{2}}\right),
$$

where $v_{\mathrm{Ti}}=\left(k_{B} T_{\mathrm{i}} / m_{\mathrm{i}}\right)^{1 / 2}$ is the ion thermal speed and $T_{\mathrm{i}}$ is the ion temperature. For frequencies near or above the lower hybrid frequency, the ion susceptibility follows approximately fluid theory,

$$
\chi_{\mathrm{i}}=-\frac{\omega_{\mathrm{pi}}^{2}}{\omega^{2}-3 v_{\mathrm{Ti}}^{2} k^{2}},
$$

where $\omega_{\mathrm{pi}}=\sqrt{4 \pi n_{0} Z_{\mathrm{i}} e^{2} / m_{\mathrm{i}}}$ is the ion plasma frequency.

The analysis uses typical parameters for the experiment, with $B_{0}=0.268 \mathrm{~T}$ and a total plasma density $n_{0}=1.70 \times$ $10^{13} \mathrm{~cm}^{-3}$ giving $\omega_{\mathrm{uh}} / \omega_{\mathrm{ce}} \approx 5.1$. The warm electron temperature is taken to be $T_{\mathrm{w}}=400 \mathrm{eV}$, and the cold electron temperature $T_{\mathrm{c}}=1 \mathrm{eV}$, such that $v_{\mathrm{Tw}}=20 v_{\mathrm{Tc}}$. The ion temperature is taken to be $T_{\mathrm{i}}=0.67 \mathrm{eV}$.

Figure 3 shows theoretical dispersion curves and growth rates together with Vlasov simulation results for a composition of a cold Maxwellian distribution function and a warm thermal distribution for a range of different values of $\eta_{\mathrm{w}}$. To obtain the complex-valued wave frequency $\widetilde{\omega}=\omega+i \gamma$ from the dispersion relation (9), where $\omega$ is the real frequency and $\gamma$ is the growth rate, the integrals over $\psi$ in Eqs. (11) and (13) are carried out numerically by means of a sum representation and the dispersion relations are solved iteratively for $\widetilde{\omega}$. The dispersion curves for the electron Bernstein modes are shown in the left column of Fig. 3 and the growth rate of unstable modes is shown in the right column. To compare with previous results $[29,30]$, the upper hybrid frequency of the cold 

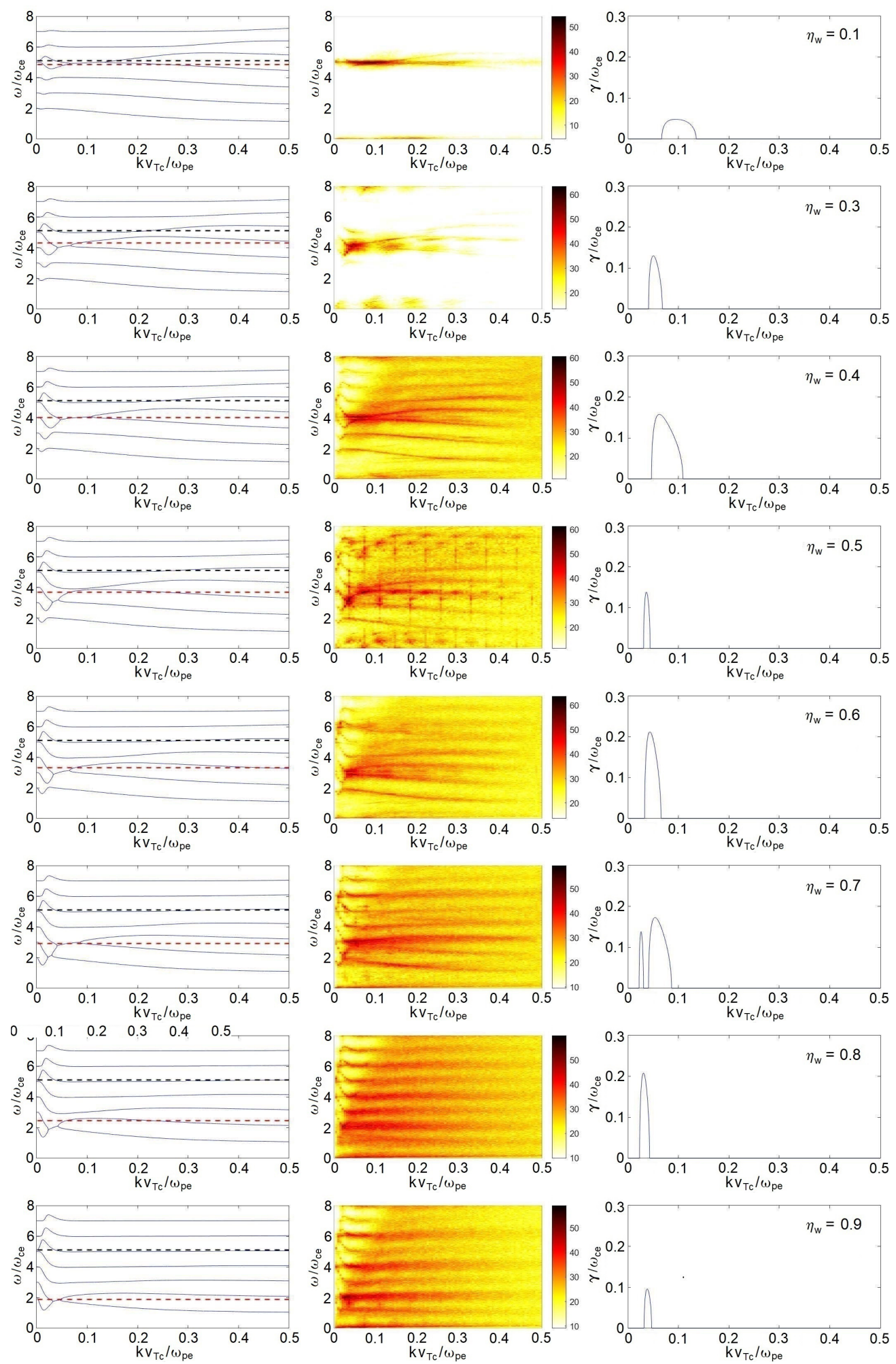

FIG. 3. Dispersion diagram (left), spatiotemporal power spectrum ( $\mathrm{dB}$, arb. units) obtained in Vlasov simulations (middle), and the instability growth rate (right) for various values of $\eta_{\mathrm{w}}=n_{\mathrm{w}} / n_{0}$; other parameters are $\omega_{\mathrm{uh}} / \omega_{\mathrm{ce}}=5.1, v_{\mathrm{Tw}}=20 v_{\mathrm{Tc}}$. In the left column, the black dashed line indicates the upper hybrid frequency $\omega_{\mathrm{uh}}$, and the red dashed line indicates the upper hybrid frequency of the cold component $\omega_{\mathrm{uh}, \mathrm{c}}=\sqrt{\omega_{\mathrm{pe}}^{2}\left(1-\eta_{\mathrm{w}}\right)+\omega_{\mathrm{ce}}^{2}}$. No instabilities are predicted for $\eta_{\mathrm{w}}=0.2$ and 1.0 (not shown). 

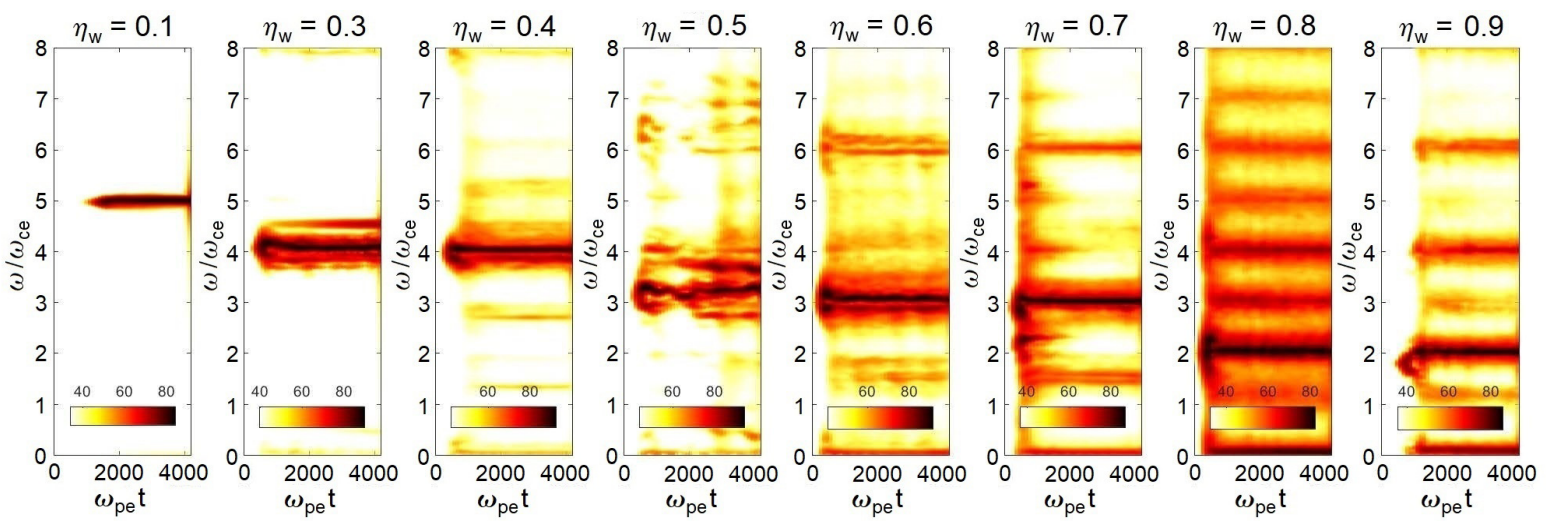

FIG. 4. Power spectrograms (dB, arb. units) obtained in Vlasov simulations for $\omega_{\mathrm{uh}} / \omega_{\mathrm{ce}}=5.1, v_{\mathrm{Tw}}=20 v_{\mathrm{Tc}}$, and different values of $\eta_{\mathrm{w}}$.

component

$$
\omega_{\mathrm{uh}, \mathrm{c}}=\sqrt{\omega_{\mathrm{pe}}^{2}\left(1-\eta_{\mathrm{w}}\right)+\omega_{\mathrm{ce}}^{2}}
$$

is indicated by horizontal dashed red lines in the left column of Fig. 3. It is seen that $\omega_{\text {uh,c }}$ coincides with or is close to the frequency of the dominant unstable modes. Instabilities take place for wave numbers where two electron Bernstein branches have merged, resulting in that the instability "jumps" to lower Bernstein modes as the value of $\eta_{\mathrm{w}}$ increases. It is noticeable that no instability takes place for a pure thermal ring distribution with $\eta_{\mathrm{w}}=1$ (not shown), confirming that the importance of the cold component in enabling the instability.

A set of Vlasov simulations was undertaken using the same sets of parameters, to check the analytic results and to study the nonlinear evolution of the system. The two-dimensional Vlasov-Boltzmann simulations use a Fourier method in velocity space $[58,59]$. The spatial simulation box is restricted to one dimension ranging from $x=0$ to $x=1200 v_{\mathrm{Tc}} / \omega_{\mathrm{pe}}$ using 200 intervals to resolve the solution, and in velocity space the maximum velocity represented in the $v_{x}$ and $v_{y}$ directions was $\pm 19 v_{\mathrm{Tc}}$ for the cold electrons, $\pm 150 v_{\mathrm{Tc}}$ for the warm electrons, and $\pm 0.38 v_{\mathrm{Tc}}$ for the ions, resolved by 120 intervals in each velocity dimension. The ions contribute primarily as a neutralizing background, and the somewhat reduced ion mass was taken to be equal to the proton mass. The theoretical results compare well with Vlasov simulations, where the wave electric field in the time $t=0$ to $4200 \omega_{\mathrm{pe}}^{-1}$ is Fourier transformed in space and time, and the resulting spatiotemporal power spectrum plotted in logarithmic (dB) scale in the middle column of Fig. 3. The equilibrium distribution functions were used as initial conditions in the simulations, where small-amplitude density perturbations $\left(\sim 10^{-5}\right.$ of the equilibrium densities) were added to seed the instability. It is seen that wave energy is concentrated at the frequencies of the electron Bernstein waves. Enhancement of the wave amplitude and widening of the spectra in frequency take place for wave numbers where there is an instability as shown in the right column of Fig. 3. For $\eta_{\mathrm{w}}=0.5$ a semiperiodic pattern can be seen as a function of wave number, which is due to a lower hybrid wave generated at the nonlinear saturation of the instability, with the lower hybrid wave having half the wavelength (twice the wave number) of the unstable electron Bernstein wave. This effect, although weaker, can be seen for other values of $\eta_{\mathrm{w}}$ as well.

In Fig. 4, spectrograms of the electric field obtained in the Vlasov simulations are constructed using a moving Gaussian window in time $\exp \left[-\left(t-t_{0}\right)^{2} / D^{2}\right]$ of width $D=525 \omega_{\mathrm{pe}}^{-1}$. The excitations in general take place for frequencies near the ones predicted by linear theory for the fastest growing modes, where the frequency of the unstable modes decreases with with the cold density fraction, following $\omega \approx \omega_{\mathrm{uh}, \mathrm{c}}$. In the nonlinear evolution of the system, spectral excitations can be seen around each cyclotron harmonic where instabilities are predicted. For $\eta_{\mathrm{w}}=0.5$ the strongest wave excitations are between the $3 \mathrm{rd}$ and 4 th cyclotron harmonic.
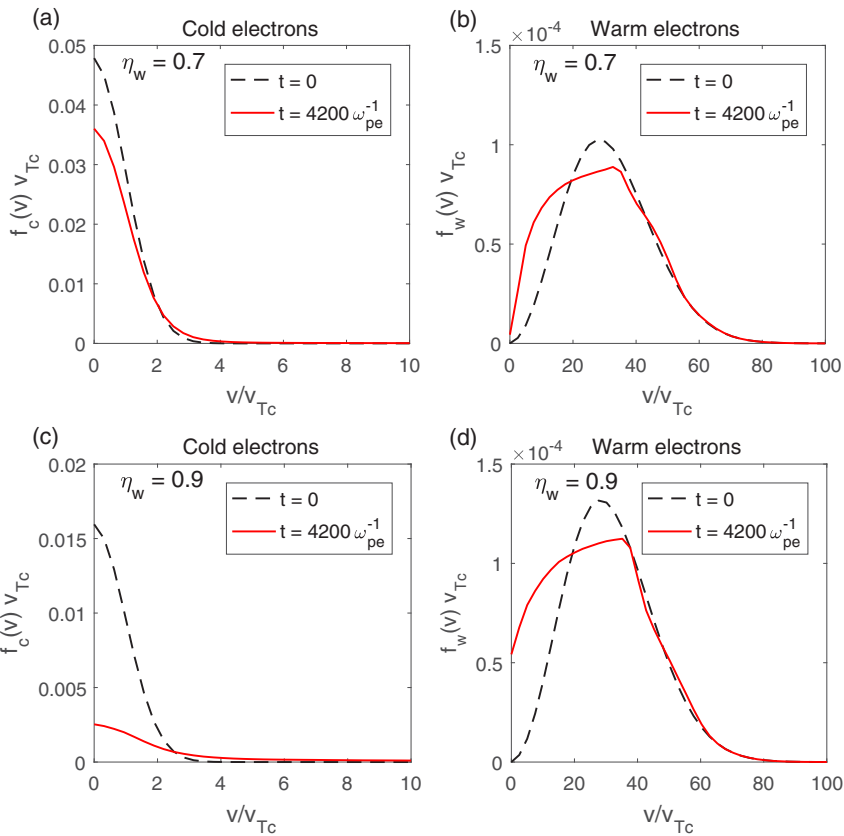

FIG. 5. The electron distribution function for cold (left) and warm (right) electrons in Vlasov simulations using $\eta_{\mathrm{w}}=0.7$ (top) and 0.9 (bottom) at $t=0$ and $4200 \omega_{\mathrm{pe}}^{-1}$. The distribution functions have been averaged over space and gyroangle. 


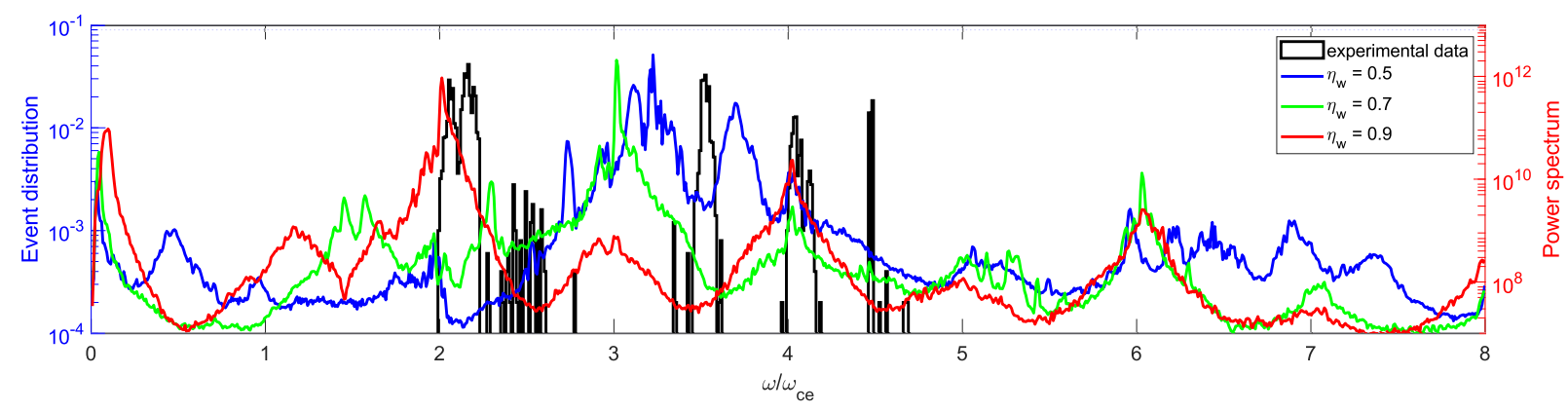

FIG. 6. Experimental data combined with Fourier spectra (arb. units) of numerically calculated wave forms for $\omega_{\mathrm{uh}} / \omega_{\mathrm{ce}}=5.1, v_{\mathrm{Tw}}=$ $20 v_{\mathrm{Tc}}$, and different values of $\eta_{\mathrm{w}}$. Excitations at $\omega \ll \omega_{\mathrm{ce}}$ are due to lower hybrid oscillations.

Figure 5 shows the distribution functions of the cold and warm electrons (averaged over space and gyroangle) initially at $t=0$ and at the end of the simulations at $t=4200 \omega_{\mathrm{pe}}^{-1}$. The free energy of the instability is in the warm ring distributed electrons leading to a positive slope in the combined electron distribution function. A fraction of the warm ring distributed electrons for velocities $v \lesssim 30 v_{\mathrm{Tc}}$ are scattered to lower velocities, converting their kinetic energy to wave energy. In the nonlinear saturation of the instability, the cold electrons are heated, in particular for $\eta_{w}=0.9$ and 0.8 , where the frequency of the unstable mode is at the second electron cyclotron harmonic (cf. Figs. 6 and 4). Through the right-hand side of Eq. (5) the ring distribution is continuously replenished giving new free energy for the instability to take place.

\section{COMPARISON WITH THE EXPERIMENTAL RESULTS}

To compare results of numerical analysis with the experimental data the experimental spectrograms are transformed to a similar format taking into account the change of ambient magnetic field with time. The spectrograms are filtered using a noise reduction algorithm. As a result, a set of spectrograms is obtained where only intense emissions are present. Next, all data points in temporally evolving spectra are normalized by the electron cyclotron frequency in the trap center at the same moment in time. Finally, these normalized spectrograms for both spectral channels are concatenated to retrieve a distribution of events in the electron cyclotron frequency domain.

Figure 6 shows integrated spectra obtained from the experimental result in Fig. 2 together with Fourier spectra of numerically calculated wave forms shown in Fig. 4. They show spectral peaks at the 2nd cyclotron harmonic, as well as between the 3rd and 4th cyclotron harmonic and between the 4th and 5th cyclotron harmonic. As mentioned above, the excitation at the 4 th cyclotron harmonic has been identified as a nonlinear harmonic of the excitation at the 2 nd cyclotron harmonic. The results are consistent with the ones seen at the cyclotron harmonics in the simulated spectrograms in Fig. 4. The excitation between the 3rd and 4th harmonic indicates that initially in the experiment the typical fraction of warm electrons is $\approx 50 \%$ with a cold fraction also about $50 \%$, while at a later time the excitation has shifted to the 2 nd cyclotron harmonic indicating an increase of the hot component to $\eta_{\mathrm{w}} \gtrsim 80 \%$ and a corresponding decrease of the cold component to a fraction $\lesssim 20 \%$.

\section{CONCLUSIONS}

Our results show that electrostatic electron cyclotron instabilities can be studied in a mirror-confined ECR discharge where a combination of warm, ring-distributed electrons and a much colder population of electrons occur naturally. The fraction of cold electrons determines the frequency of the unstable modes. The experiment shows features in the radiated spectra that are consistent with an early-phase plasma having a composition of about $50 \%$ each of warm and cold electrons radiating primarily between the 3rd and 4th cyclotron harmonics, evolving with time toward an admixture of $80 \%$ warm and $20 \%$ cold electrons radiating predominately near the 2nd cyclotron harmonic. Future experiments will focus on more precise measurements of the plasma density which will give the ratio $\omega_{\mathrm{uh}} / \omega_{\mathrm{ce}}$ more accurately, and on the measurements of the cold, warm, and hot components of the electron distribution function. The process of plasma wave transformation from the electrostatic electron Bernstein waves into electromagnetic emissions, experimentally registered outside the plasma volume, will be discussed in future works. An interesting application of the laboratory experiments is to space plasma where multicomponent electron distributions exist [30], to understand the banded emissions frequently recorded by spacecraft near the Earth [13-18] and other planets [19-22].

Data supporting the figures can be found in Ref. [60].

\section{ACKNOWLEDGMENTS}

The authors gratefully acknowledge the funders of the research. In Russia the project is funded by the RFBR and K under Project No. 19-52-10007, and by the Grants Council of the President of the Russian Federation (Grant No. MK-2593.2019.2). In the UK the project is enabled by funding from the Royal Society, Award No. IEC/R2/181158, and the UK EPSRC under Grants No. EP/R034737/1, No. EP/R004773/1, No. EP/M009386/1, and No. EP/G04239X/1. 
[1] L. B. Wilson, III, D. G. Sibeck, A. W. Breneman, O. L. Contel, C. Cully, D. L. Turner, V. Angelopoulos, and D. M. Malaspina, Quantified energy dissipation rates in the terrestrial bow shock: 2. Waves and dissipation, J. Geophys. Res.: Space Phys. 119, 6475 (2014).

[2] S. P. Gary and J. Wang, Whistler instability: Electron anisotropy upper bound, J. Geophys. Res.: Space Phys. 101, 10749 (1996).

[3] Š. Stverák, P. Trávnícek, M. Maksimovic, E. Marsch, A. N. Fazakerley, and E. E. Scime, Electron temperature anisotropy constraints in the solar wind, J. Geophys. Res.: Space Phys. 113, A03103 (2008).

[4] R. Bingham and R. A. Cairns, Generation of auroral kilometric radiation by electron horseshoe distributions, Phys. Plasmas 7 , 3089 (2000).

[5] D. B. Melrose and G. A. Dulk, Electron-cyclotron masers as the source of certain solar and stellar radio bursts, Astrophys. J. 259, 844 (1982).

[6] D. B. Melrose, Coherent emission mechanisms in astrophysical plasmas, Rev. Mod. Plasma Phys. 1, 5 (2017).

[7] A. G. Demekhov and V. Y. Trakhtengerts, A mechanism of formation of pulsating aurorae, J. Geophys. Res.: Space Phys. 99, 5831 (1994).

[8] S. P. Gary and I. H. Cairns, Electron temperature anisotropy instabilities: Whistler, electrostatic and z mode, J. Geophys. Res.: Space Phys. 104, 19835 (1999).

[9] B. Eliasson and M. Lazar, Nonlinear evolution of the electromagnetic electron-cyclotron instability in bi-Kappa distributed plasma, Phys. Plasmas 22, 062109 (2015).

[10] N. P. Meredith, R. B. Horne, R. M. Thorne, and R. R. Anderson, Survey of upper band chorus and ECH waves: Implications for the diffuse aurora, J. Geophys. Res.: Space Phys. 114, A07218 (2009).

[11] Y. Wu, X. Tao, X. Liu, L. Chen, H. Xie, K. Liu, and R. B. Horne, Particle-in-cell simulation of electron cyclotron harmonic waves driven by a loss cone distribution, Geophys. Res. Lett. 47, e2020GL087649 (2020).

[12] A. K. Tripathi, R. P. Singhal, and O. N. Singh, II, The generation of Saturn's aurora at lower latitudes by electrostatic waves, J. Geophys. Res.: Space Phys. 123, 3565 (2018).

[13] A. W. Breneman, C. A. Cattell, K. Kersten, A. Paradise, S. Schreiner, P. J. Kellogg, K. Goetz, and L. B. Wilson, III, STEREO and Wind observations of intense cyclotron harmonic waves at the Earth's bow shock and inside the magnetosheath, J. Geophys. Res.: Space Phys. 118, 7654 (2013).

[14] K. A. Goodrich, R. Ergun, S. J. Schwartz, L. B. Wilson, III, D. Newman, F. D. Wilder, J. Holmes, A. Johlander, J. Burch, R. Torbert, Y. Khotyaintsev, P.-A. Lindqvist, R. Strangeway, C. Russell, D. Gershman, B. Giles, and L. Andersson, MMS observations of electrostatic waves in an oblique shock crossing, J. Geophys. Res.: Space Phys. 123, 9430 (2018).

[15] W. Li, D. Graham, Y. Khotyaintsev, A. Vaivads, M. André, K. Min, K. Liu, B. B. Tang, C. Wang, K. Fujimoto, C. Norgren, S. Toledo-Redondo, P.-A. Lindqvist, R. E. Ergun, R. B. Torbert, A. C. Rager, J. C. Dorelli, D. J. Gershman, B. L. Giles, B. Lavraud, F. Plaschke, W. Magnes, O. L. Contel, C. T. Russell, and J. L. Burch, Electron Bernstein waves driven by electron crescents near the electron diffusion region, Nat. Commun. 11, 141 (2020).
[16] J. D. Menietti and P. H. Yoon, Plasma waves and fine structure emission bands within a plasmapause density cavity source region, Geophys. Res. Lett. 33, L15101 (2006).

[17] A. Shinbori, T. Ono, M. Iizima, A. Kumamoto, S. Shirai, A Hanaoka, K. Okamoto, M. Ohashi, and H. Oya, Electrostatic electron cyclotron harmonic waves observed by the Akebono satellite near the equatorial region of the plasmasphere, Earth, Planets Space 59, 613 (2007).

[18] F. El-Lemdani Mazouz, J.-L. Rauch, P. Décréau, J.-G. Trotignon, X. Vallières, F. Darrouzet, P. Canu, and X. Suraud, Wave emissions at half electron gyroharmonics in the equatorial plasmasphere region: CLUSTER observations and statistics, Adv. Space Res. 43, 253 (2009).

[19] W. S. Kurth, D. D. Barbosa, D. A. Gurnett, and F. L. Scarf, Electrostatic waves in the Jovian magnetosphere, Geophy. Res. Lett. 7, 57 (1980).

[20] W. S. Kurth, G. B. Hospodarsky, D. L. Kirchner, B. T. Mokrzycki, T. F. Averkamp, W. T. Robison, C. W. Piker, M. Sampl, and P. Zarka, The Juno waves investigation, Space Sci. Rev. 213, 347 (2017).

[21] X. Tao, R. M. Thorne, R. B. Horne, S. Grimald, C. S. Arridge, G. B. Hospodarsky, D. A. Gurnett, A. J. Coates, and F. J. Crary, Excitation of electron cyclotron harmonic waves in the inner Saturn magnetosphere within local plasma injections, J. Geophys. Res.: Space Phys. 115, A12204 (2010).

[22] J. D. Menietti, T. F. Averkamp, W. S. Kurth, S.-Y. Ye, D. A. Gurnett, and B. Cecconi, Survey of Saturn electrostatic cyclotron harmonic wave intensity, J. Geophys. Res.: Space Phys. 122, 8214 (2017).

[23] R. A. Dory, G. E. Guest, and E. G. Harris, Unstable Electrostatic Plasma Waves Propagating Perpendicular to a Magnetic Field, Phys. Rev. Lett. 14, 131 (1965).

[24] F. W. Crawford and J. A. Tataronis, Absolute instabilities of perpendicularly propagating cyclotron harmonic plasma waves, J. Appl. Phys. 36, 2930 (1965).

[25] R. W. Fredricks, Plasma instability at $(n+1 / 2) f_{c}$ and its relationship to some satellite observations, J. Geophys. Res. 76, 5344 (1971).

[26] V. I. Karpman, J. K. Alekhin, N. D. Borisov, and N. A. Rjabova, Electrostatic electron-cyclotron waves in plasma with a losscone distribution, Plasma Phys. 17, 361 (1975).

[27] P. H. Yoon, F. Hadi, and A. Qamar, Bernstein instability driven by thermal ring distribution, Phys. Plasmas 21, 074502 (2014).

[28] T. S. T. Young, J. D. Callen, and J. E. McCune, High-frequency electrostatic waves in the magnetosphere, J. Geophys. Res. 78, 1082 (1973).

[29] R. F. Hubbard and T. J. Birmingham, Electrostatic emissions between electron gyroharmonics in the outer magnetosphere, J. Geophys. Res.: Space Phys. 83, 4837 (1978).

[30] K. Rönnmark, H. Borg, P. J. Christiansen, M. P. Gough, and D. Jones, Banded electron cyclotron harmonic instability-a first comparison of theory and experiment, Space Sci. Rev. 22, 401 (1978).

[31] J. A. Byers and M. Grewal, Perpendicularly propagating plasma cyclotron instabilities simulated with a one-dimensional computer model, Phys. Fluids 13, 1819 (1970).

[32] M. Ashour-Abdalla, J. N. Leboeuf, J. M. Dawson, and C. F. Kennel, A simulation study of cold electron heating by loss cone instabilities, Geophys. Res. Lett. 7, 889 (1980). 
[33] J. M. Dawson, Particle simulation of plasmas, Rev. Mod. Phys. 55, 403 (1983).

[34] M. Horký and Y. Omura, Novel nonlinear mechanism of the generation of non-thermal continuum radiation, Phys. Plasmas 26, 022904 (2019).

[35] K. Ronald, D. C. Speirs, S. L. McConville, A. D. R. Phelps, C. W. Robertson, C. G. Whyte, W. He, K. M. Gillespie, A. W. Cross, and R. Bingham, Radio frequency resonator structure and diagnostic measurements for a laboratory simulation of auroral kilometric radiation, Phys. Plasmas 15, 056503 (2008).

[36] R. Bingham, D. C. Speirs, B. J. Kellett, I. Vorgul, S. L. McConville, R. A. Cairns, A. W. Cross, A. D. R. Phelps, and K. Ronald, Laboratory astrophysics: Investigation of planetary and astrophysical maser emission, Space Sci. Rev. 178, 695 (2013).

[37] D. C. Speirs, R. Bingham, R. A. Cairns, I. Vorgul, B. J. Kellett, A. D. R. Phelps, and K. Ronald, Backward Wave CyclotronMaser Emission in the Auroral Magnetosphere, Phys. Rev. Lett. 113, 155002 (2014).

[38] J. M. Urrutia and R. L. Stenzel, Observations of odd-half cyclotron harmonic emissions in a shell-Maxwellian laboratory plasma, J. Geophys. Res.: Space Phys. 88, 7086 (1983).

[39] C. Krafft, G. Matthieussent, P. Thevenet, and G. Belmont, Simultaneous emissions at $n \omega_{c}$ and $(n+1 / 2) \omega_{c}$ in space and laboratory plasmas, J. Geophys. Res.: Space Phys. 97, 14977 (1992).

[40] J. P. Tate and C. B. Wharton, Radio-frequency excitation of harmonic microwave radiation from a Penning reflex discharge, Phys. Fluids B: Plasma Phys. 5, 1358 (1993).

[41] R. L. Stenzel and G. Golubyatnikov, Cyclotron harmonic lines in the thermal magnetic fluctuation spectrum of spiraling electrons in plasmas, Phys. Fluids B: Plasma Phys. 5, 3789 (1993).

[42] M. V. Starodubtsev, A. V. Kostrov, V. V. Nazarov, and G. V. Permitin, Excitation of quasielectrostatic waves in a laboratory magnetoplasma with weak spatial dispersion, Phys. Rev. E 72, 026401 (2005).

[43] A. V. Vodop'yanov, S. V. Golubev, V. G. Zorin, S. V. Razin, and M. A. Shilov, Plasma parameters of an electron cyclotron resonance discharge in a magnetic mirror in a quasi-gasdynamic confinement regime, Tech. Phys. Lett. 25, 588 (1999).

[44] A. G. Shalashov, M. E. Viktorov, D. A. Mansfeld, and S. V. Golubev, Kinetic instabilities in a mirror-confined plasma sustained by high-power microwave radiation, Phys. Plasmas 24, 032111 (2017).

[45] A. G. Shalashov, E. D. Gospodchikov, and I. V. Izotov, Electron-cyclotron heating and kinetic instabilities of a mirrorconfined plasma: The quasilinear theory revised, Plasma Phys. Control. Fusion 62, 065005 (2020).

[46] I. Izotov, O. Tarvainen, V. Skalyga, D. Mansfeld, T. Kalvas, H. Koivisto, and R. Kronholm, Measurement of the energy distribution of electrons escaping minimum-B ECR plasmas, Plasma Sources Sci. Technol. 27, 025012 (2018).
[47] S. V. Golubev, I. V. Izotov, D. A. Mansfeld, and V. E. Semenov, Experimental electron energy distribution function investigation at initial stage of electron cyclotron resonance discharge, Rev. Sci. Instrum. 83, 02B504 (2012).

[48] M. E. Viktorov, A. G. Shalashov, D. A. Mansfeld, and S. V. Golubev, Observation of quasi-periodic frequency sweeping in electron cyclotron emission of nonequilibrium mirror-confined plasma, Europhys. Lett. 116, 55001 (2016).

[49] A. A. Skovoroda and A. V. Timofeev, Investigation of ECR discharge within the baseball and cusp geometry with longitudinal input of gyrotron power, in Proceedings 2nd International Workshop, Strong Microwaves in Plasmas, Vol. 1, edited by A. G. Litvak (Institute of Applied Physics, Nizhny Novgorod, 1994), pp. 328-346.

[50] D. Forslund, R. Morse, C. Nielson, and J. Fu, Electron cyclotron drift instability and turbulence, Phys. Fluids 15, 1303 (1972).

[51] M. E. Viktorov, A. G. Shalashov, E. D. Gospodchikov, N. Y. Semin, and S. V. Golubev, Zebra-like patterns in whistler wave emission spectra from nonequilibrium mirror-confined laboratory plasma, Phys. Plasmas 27, 062104 (2020).

[52] A. V. Vodop'yanov, S. V. Golubev, A. G. Demekhov, V. G. Zorin, D. A. Mansfel'd, S. V. Razin, and V. Y. Trakhtengerts, Laboratory modeling of nonstationary processes in space cyclotron masers: First results and prospects, Plasma Phys. Rep. 31, 927 (2005).

[53] R. J. Goldston and P. H. Rutherford, Introduction to Plasma Physics, Plasma Physics Series (Institute of Physics Publishing, 1995).

[54] S. V. Golubev, S. V. Razin, V. E. Semenov, A. N. Smirnov, A. V. Vodopyanov, and V. G. Zorin, Formation of multi-charged ions and plasma stability at quasigasdynamic plasma confinement in a mirror magnetic trap, Rev. Sci. Instrum. 71, 669 (2000).

[55] V. Skalyga, I. Izotov, S. Golubev, A. Sidorov, S. Razin, A. Vodopyanov, O. Tarvainen, H. Koivisto, and T. Kalvas, New progress of high current gasdynamic ion source (invited), Rev. Sci. Instrum. 87, 02A716 (2016).

[56] V. V. Zheleznyakov and E. Y. Zlotnik, Cyclotron wave instability in the corona and origin of solar radio emission with fine structure. I. Bernstein modes and plasma waves in a hybrid band, Solar Phys. 43, 431 (1975).

[57] S. M. Grach, On kinetic effects in the ionospheric $F$-region modified by powerful radio waves, Radiophys. Quantum Electron. 42, 572 (1999).

[58] B. Eliasson, D. C. Speirs, and L. K. S. Daldorff, Electrostatic electron cyclotron instabilities near the upper hybrid layer due to electron ring distributions, Plasma Phys. Control. Fusion 58, 095002 (2016).

[59] B. Eliasson, Numerical simulations of the Fourier-transformed Vlasov-Maxwell system in higher dimensions - theory and applications, Transport Theory Stat. Phys. 39, 387 (2010).

[60] https://doi.org/10.15129/45a4eb8b-4812-49ee-bf5e1 ba22b603690. 